\title{
Erratum to: Genetic Counselling for Maternally Inherited Mitochondrial Disorders
}

\author{
Joanna Poulton ${ }^{1} \cdot$ Josef Finsterer $^{2}$ Patrick Yu-Wai-Man ${ }^{3,4,5,6}$
}

Published online: 4 July 2017

(c) Springer International Publishing AG 2017

\section{Erratum to: Mol Diagn Ther DOI 10.1007/s40291-017-0279-7}

Errors were subsequently identified in the article, and the following corrections should be noted:

In Section 3.2.2.1, paragraph 1, lines 185-192: The following three sentences, which previously read:

"Compared with mtDNA point mutations, single largescale mtDNA deletions usually represent de novo mutational events occurring either in an oocyte or during embryogenesis [5]. Therefore, only the proband is usually

The online version of the original article can be found under doi:10.1007/s40291-017-0279-7.

Josef Finsterer

fifigs1@yahoo.de

Joanna Poulton

joanna.poulton@obs-gyn.ox.ac.uk

Patrick Yu-Wai-Man

py237@cam.ac.uk

1 Nuffield Department of Obstetrics and Gynaecology, University of Oxford, Oxford, UK

2 Krankenanstalt Rudolfstiftung, Postfach 20, 1180 Vienna, Austria

3 Wellcome Trust Centre for Mitochondrial Research, Institute of Genetic Medicine, Newcastle University,

Newcastle upon Tyne, UK

4 Newcastle Eye Centre, Royal Victoria Infirmary, Newcastle upon Tyne, UK

5 NIHR Biomedical Research Centre, Moorfields Eye Hospital and UCL Institute of Ophthalmology, London, UK

6 Department of Clinical Neurosciences, School of Clinical Medicine, University of Cambridge, Cambridge, UK affected in the family, but there is nevertheless a low risk of transmission by the mother of an affected child. The recurrence risk for a single, large-scale mtDNA deletion has been estimated at"

should read:

"Compared with mtDNA point mutations, single largescale mtDNA deletions are usually sporadic. Therefore, only the proband is usually affected in the family. There is nevertheless a low risk of transmission by the mother of an affected child with the estimated recurrence risk for a single large-scale mtDNA deletion being 1 in 117 ( 4\%)"

In Section 3.2.2.2, paragraph 1, lines 200-214: The following paragraph, which previously read:

\section{“3.2.2.2 Insertions, Duplications and Triplications}

Pathogenic mtDNA insertions, duplications or triplications have been occasionally reported as causing MIDs, either as large-scale [23] or small-scale [24] rearrangements. In a particular case with maternally inherited diabetes and deafness (MIDD) and complex-I deficiency in fibroblasts, mtDNA sequencing revealed a heteroplasmic insertion of one or two cytosine residues in the coding region of the MT-ND6 gene (m.14535_14536insC or CC), leading to a premature stop codon [25]. A 9-bp triplication in the MT-CYTB gene was detected in an African patient with isolated mitochondrial myopathy [24]. In a patient with dilated cardiomyopathy, the causative mutation was a 15 bp duplication in the $t R N A^{\text {Pro }}$ gene [26]."

should read:

\section{“3.2.2.2. Insertions and Duplications}

Pathogenic mtDNA insertions, largely duplications, have been occasionally reported as polymorphisms and as 
causing MIDs. The latter are either large-scale [23] or small-scale [24] rearrangements. Unlike the majority of mtDNA rearrangements they can be maternally inherited [25]. While the phenotype can be classical Kearns-Sayre syndrome, endocrinopathies are common and families may manifest maternally inherited diabetes and deafness (MIDD). In one family with MIDD associated with a complex 1 deficiency in fibroblasts, mtDNA sequencing revealed a heteroplasmic insertion of one or two cytosine residues in the coding region of the MT-ND6 gene (m.14535_14536insC or CC), leading to a premature stop codon [26]."

In Section, Compliance with Ethical Standards, Funding, lines 732-740: The following three sentences, which previously read:

"PYWM is supported by a Clinician Scientist Fellowship Award (G1002570) from the Medical Research Council (MRC) (UK). PYWM also receives funding from Fight for Sight (UK), the UK National Institute of Health Research (NIHR) as part of the Rare Diseases Translational Research Collaboration, and the NIHR Biomedical Research Centre based at Moorfields Eye Hospital NHS Foundation Trust and UCL Institute of Ophthalmology. The views expressed are those of the author(s) and not necessarily those of the NHS, the NIHR or the Department of Health."

should read:

"JP is supported by the Lily Foundation, the New Life Charity for Disabled Children and the Angus Memorial Mitochondrial Fund. She acknowledges salary support from the NHS Highly Specialised Rare Mitochondrial Disorders of Adults and Children Service. PYWM is supported by a Clinician Scientist Fellowship Award (G1002570) from the Medical Research Council (MRC) (UK). PYWM also receives funding from Fight for Sight
(UK), the UK National Institute of Health Research (NIHR) as part of the Rare Diseases Translational Research Collaboration, and the NIHR Biomedical Research Centre based at Moorfields Eye Hospital NHS Foundation Trust and UCL Institute of Ophthalmology. The views expressed are those of the author(s) and not necessarily those of the NHS, the NIHR or the Department of Health."

\section{Correction in the reference list}

Reference list: References 24-26, which previously read:

24. Mkaouar-Rebai E, Felhi R, Tabebi M, Alila-Fersi O, Chamkha I, Maalej M, et al. Mitochondrial DNA triplication and punctual mutations in patients with mitochondrial neuromuscular disorders. Biochem Biophys Res Commun. 2016;473:578-85.

25. Bannwarth S, Abbassi M, Valéro R, Fragaki K, Dubois N, Vialettes B, et al. A novel unstable mutation in mitochondrial DNA responsible for maternally inherited diabetes and deafness. Diabetes Care. 2011;34:2591-3.

26. Cardena MM, Mansur AJ, Pereira Ada C, Fridman C. A new duplication in the mitochondrially encoded tRNA proline gene in a patient with dilated cardiomyopathy. Mitochondrial DNA. 2013;24:46-9.

should read:

24. Bannwarth S, Abbassi M, Valéro R, Fragaki K, Dubois N, Vialettes B, et al. A novel unstable mutation in mitochondrial DNA responsible for maternally inherited diabetes and deafness. Diabetes Care. 2011;34:2591-3.

25. Cardena MM, Mansur AJ, Pereira Ada C, Fridman C. A new duplication in the mitochondrially encoded tRNA proline gene in a patient with dilated cardiomyopathy. Mitochondrial DNA. 2013;24:46-9.

26. Poulton J, Holt I. Mitochondrial DNA: does more lead to less? Nat Genet. 1994;8:313-5. 